\title{
Overview for special issue: global issues in clinical engineering
}

\author{
Yadin David $^{1} \cdot$ Ernesto ladanza ${ }^{2}$ (1) \\ Published online: 11 February 2020 \\ (C) IUPESM and Springer-Verlag GmbH Germany, part of Springer Nature 2020
}

Keywords Clinical engineering $\cdot$ Technology $\cdot$ Patient $\cdot$ Healthcare $\cdot$ Outcomes $\cdot$ Safety $\cdot$ Quality $\cdot$ Education $\cdot$ Modeling $\cdot$ Device

There is copious satisfaction in serving as Co-Editor of a Special Issue especially as it focuses on international Clinical Engineering subjects. Over the past few years, with Ernesto Iadanza and Luis Kun, we worked together on identification of subjects that clinical engineers around the world face and we assembled collection of manuscripts that explore them representing the four corners of the world. A world that consumes and dependent upon healthcare services literally everywhere from the Antarctica to the Everest as well as in outer space. The development and provision of these healthcare services would have been impossible without the provision of safe and reliable technology. Healthcare services and technology are in a dependent relationship that is at all time high with every indicator suggesting further future increase of this trend. It is, therefore, important to recognize the need for methodology to manage the whole life cycle of the technology in healthcare and the critical role of clinical engineers in delivering the unique expertise to properly deploy and manage it.

This special issue is in some way serving as response for my clinical engineering colleagues who have continuously, but correctly, expressed their frustration with the inability they face in having their work published in scientific literature. The benefits from knowledge sharing have been previously established and is serving as the foundation for every profession, including Clinical Engineering, to deliver positive impact on outcomes (in our case on people health and wellness) and satisfaction with their contribution. While Clinical

\footnotetext{
Yadin David

David@biomedeng.com

Ernesto Iadanza

ernesto.iadanza@unifi.it

1 Global Clinical Engineering Journal, 1111 Hermann Drive, Suite 12B, Houston, TX 77004, USA

2 Chair, Health Technology Assessment Division of the International Federation for Medical and Biological Engineering, Florence, Italy
}

Engineering profession is not ubiquitous nor known field, its impact is critical for delivering successful and intended outcomes as the articles in this issue show.

This special issue is composed of several articles presenting varied challenges ranging in topics from technology adoption pathway [1] and mathematical modeling of manpower capacity [2] and system for supporting clinical decision [3] to computerized maintenance in low resources countries [4] and mobility associated with wireless issues as well as Evidence-Based Impact by Clinical Engineers on Global Patients Outcomes [5]. Our ability to meet these challenges depends on, to a large degree, the preparation of competent manpower capacity that is able to deliver safety and quality services as describes in the paper on Mathematical Modeling of Clinical Engineering [2]. Similarly, to the expanded roles described in the paper on Clinical Engineering Advances in Argentina. [6] These topics are part of the stages of the technology life cycle. Life cycle that starts with innovation, development and prototype testing and continues with compliance, commissioning, performance assurance, upgrade and end with disposition [7]. In each of these stages the focus of clinical engineer role may vary but his/her competencies are shared [8].

It has always been clear to me, that a professional may spend career in field of practice because of choices made, aspiration followed, experience with or due to failing in another. I have been working in variety of jobs ranging from design of space orbiter systems to development of smart projectiles, even served as operating theater housekeeper. Before attending college, I also worked as electronic technician and audio players repairman. All the jobs fitted into the stage of life I was in at the time. Throughout all of these experiences I never anticipated the rewards experienced from life-long engagement in the Clinical Engineering practice. A practice that turns work into mission that diligently seeks fulfillment of obligations to support better patients' outcomes, safer and more affordable and appropriate technological solutions. Practice that offers opportunities to become member of the 
special team of care providers where dependence on technology to collect, treat and make critical decisions is growing. Dependence that our own family's health outcomes may someday be impacted by. The knowledge of making a difference and finding solution to problems by applying engineering expertise and leadership are unique to $\mathrm{CE}$ as is the satisfaction from achieving that. We wanted in this issue to identify gaps and grow our professional capacity to address them, reaching more affordable and effective technological solutions to clinical challenges. It is our hope that this special issue will serve as a catalyst for further opportunities for knowledge sharing in the Clinical Engineering field as well as facilitator for future clinical engineers to join in the satisfaction of being part of unique profession. I am sure that you too will agree with me after reading the issue.

Yadin David

When Dr. Luis Kun first asked me to serve as guest editor for a special issue on clinical engineering, I immediately accepted with enthusiasm. Having a co-editor such Dr. Yadin David was both a great privilege and a guarantee of involving the best professionals in the world.

I have been serving as member of the Clinical Engineering Division Board of the International Federation for Medical and Biological Engineering (IFMBE/CED) for many years and I had the privilege of leading this board as elected chairman for three years (2015-18). That experience has given me a tremendous opportunity to increase my awareness on this very particular field of knowledge, by understanding the several challenges among different countries and settings in the world, as well as the many similar issues that clinical engineers from all over the world are facing in their daily activities.

Having the chance to interact with hundreds of colleagues from all over the world, I realized that, in comparison to the past, there is today a lot of research in clinical engineering. Methodologies for investments planning, for assessing risks, for designing management protocols based on evidence (Evidence Based Maintenance) need to be shared, discussed, criticized, tested, compared. More and more, clinical engineers are becoming aware of this and are increasing their publications on conference proceedings, new international handbooks and journals.

This special issue focusing on global issues in clinical engineering is an excellent step forward in this direction, with several articles reporting experiences from Asia, Europe, America and Africa, together with an article dedicated to a worldwide clinical engineering survey.

Many topics are discussed, including the quality of patient care, clinical decision support systems, disaster preparedness, computerized management systems, and more.

I sincerely hope you will find many answers to your questions but also - maybe more importantly - many new questions that urge answers from you!

Ernesto Iadanza

\section{Compliance with ethical standards}

Conflict of interest The authors declare that they have no conflict of interest.

Ethical approval This article does not contain any studies with human participants or animals performed by any of the authors.

\section{References}

1. Clark D, Dean G, Bolton S, Beeson B (2019) Bench to bedside: the technology adoption pathway in healthcare. Health Technol (Berl) 19.

2. Hossain MA, Ahmad M, Islam MR, David Y (2019) Mathematical modeling of clinical engineering approach to evaluate the quality of patient care. Health Technol (Berl) 1-15.

3. Iadanza E, Mudura V, Melillo P, Gherardelli M (2019) An automatic system supporting clinical decision for chronic obstructive pulmonary disease. Health Technol (Berl) 1-12.

4. Medenou D, Fagbemi LA, Houessouvo RC, et al. Medical devices in sub-Saharan Africa: optimal assistance via a computerized maintenance management system (CMMS) in Benin. Health Technol (Berl). 2019;9:219-32.

5. David Y, Judd T (2019) Evidence-based impact by clinical engineers on global patients outcomes. Health Technol (Berl) 1-19.

6. Sardá EF, Lencina M, Giles G (2019) Clinical engineering advances in Argentina. Health Technol (Berl) 1-3.

7. Wang L, Wang L, Zheng Y, Chen Y, Lu H, Chu C, Li B (2019) Establishment and practice of 5 layers guarantee system for CT/ MR equipment quality management based on multi-party cooperation. Health Technol (Berl) 1-7.

8. Tarazona RR, David Y (2019) Strengthen disasters preparedness within clinical engineering-CE expertise and knowledge. Health Technol (Berl) 1-6.

Publisher's note Springer Nature remains neutral with regard to jurisdictional claims in published maps and institutional affiliations. 